\title{
Recreando aprendizajes alternativos desde un contexto sociocultural
}

\author{
Recreating Alternative Learnings from a sociocultural context
}

\author{
Angélica Leonor Ruiz Calderón'
}

\section{Resumen}

Se ha abordado la recreación de aprendizajes alternativos hacia una complejidad holística y etológica. La metodología fue hermenéutica a textos educativos con el enfoque hacia una praxis de amor que es guía en el pensar y actuar. Esta educación promovería la solución actual para la paz, la democracia y el respeto a los derechos humanos, por lo que es necesario hacer un abordaje desde el cuestionamiento ¿cómo recreo aprendizajes alternativos?

Palabras clave: recreación de aprendizajes alternativos; complejidad holística; complejidad etológica.

\section{Summary}

The recreation of alternative learning towards a holistic and ethological complexity has been addressed. The methodology was hermeneutic to educational texts with a focus on a praxis of love that is a guide in thinking and acting. This education would promote the current solution for peace, democracy and respect for human rights, so it is necessary to approach from the questioning how do I recreate alternative learning?

Key Words: Recreation of alternative learnings; holistic complexity; ethological complexity.

\section{Introducción}

El presente artículo de revisión bibliográfica se ha enfocado en la reflexión sobre recreación de aprendizajes alternativos con referentes teóricos del paradigma emergente de la educación holística, flexible, ecológica y compleja. Darnos cuenta cómo desde nuestras experiencias de vida se pueden dar aprendizajes alternativos, soñamos con un camino de sabiduría, amor y compasión que nos conduzca a un cambio, en nuestro pensar y actuar.

En este mundo cambiante con modelos educativos de forma permanente, es fundamental considerar al aprendizaje como una construcción de significados. Esta construcción es activa, mediada y autorregulada. El aprendizaje se alcanza a través de un conjunto de procesos y habilidades cognitivas que orientan el desarrollo del pensamiento humano, en este sentido hacer que éste, no sólo se comprenda, sino también que se cree, recree y sienta, de esta manera propiciar la búsqueda del bienestar de la persona como par-

Doctora en Educación con especialidad en Mediación Pedagógica. Vicerrectora de la Universidad de las Regiones Autónomas de la Costa Caribe Nicaragüense-Recinto Universitario Las Minas. Correo: leonor.ruiz@uraccan.edu.ni

Recibido: 23/10/2017 Aprobado: 23/11/2017 
te de un grupo social, lo que implica aprendizajes recíprocos que también fortalezca la identidad personal y grupal.

Como seres sociales, tenemos la posibilidad y la responsabilidad de promover en la educación, actos reflexivos y conscientes como parte de la construcción social que se nos exige la realidad humana. Vivimos en consciencia y autosconcienca desde el lenguaje y el lenguajear, lo que implica que en los diferentes ámbitos y momentos, intencionales o no de aprendizaje, se pueden promover espacios como vía para la reflexión, donde juega una gran importancia la comunicación, especialmente la consciencia y legitimidad del otro.

En la búsqueda de recrear aprendizajes, es ineludible desprenderse de viejas ideas, costumbres, no pueden crearse cosas nuevas sino abrimos espacios para ellas. Es hora de permitirnos desprender algunas prácticas individualistas y asumir compromisos colectivos que contribuyan a la apertura, libertad, respeto, solidaridad, tolerancia y cuidado, asumiendo una actitud proactiva y de concientización de un amplio sentido responsabilidad de su accionar frente a los desafíos que enfrenta para ser partícipe en el cambio de nuestro futuro para un "mejor vivir". En este sentido, son fundamentales las relaciones éticas, que como asegura Maturana (1996), nos hace ver al otro como legitimo otro. No podemos sembrar, si la semilla no está en nosotros. Esto lo refiere Payan, (2001), acerca de las rupturas a nivel institucional que nos obliga a pasar de una realidad fragmentada y en especialidades hacia una realidad tejida entre relaciones de complejidades que nos permiten ver esa quinta dimensión que nos une con el universo.

Estas reflexiones, conducen al esperanzador imaginario de una sociedad con un sistema educativo y un modelo de desarrollo basado en el respeto y descubrimiento del sentido de la vida gracias a ese potencial de la capacidad biológica de amar y entender el fundamento científico en que se basan maestros como Maturana y Varela en relación a esa capacidad de transformación en la convivencia. Esta educación promovería la solución actual para la paz, la democracia y el respeto a los derechos humanos. Por lo que es necesario hacer un abordaje desde el cuestionamiento ¿cómo recreo aprendizajes alternativos?

\section{Material y métodos}


Es una crisis alimentada por la codicia desenfrenada y febril de minorías, que en el mundo entero han puesto en jaque a toda una sociedad, provocando la más descarnada marginalidad y pobreza; déficit alimentaria y precaria atención a la salud, la vivienda con énfasis en el deterioro y acceso a la educación. Al respecto, Leonardo Boff (2000), plantea que la falta de justicia social, urge del despertar de una nueva consciencia por el cuidado, la solidaridad, la ternura y compasión para compartir la vida y los bienes de la naturaleza; creando nuevas estructuras socioeconómicas, políticas y espirituales para la sostenibilidad de la especie y el equilibrio de la misma en el plano del paradigma holista. En este espejo transparente de crisis vemos reflejada la dura realidad vivencial de nuestra Región Autónoma de la Costa Caribe Nicaragüense.

No podemos seguir siendo cómplices de la conducción de un sistema educativo sin sentido en nuestras vidas, sin la pasión por admirar la belleza de nuestros paisajes, sin despertar sensibilidad por los colores, sonrisas, gestos, ritmos, etc, que la visión tradicional han invisibilizado y menospreciado la cultura propia de nuestros pueblos. En nuestro entorno inmediato comunitario, el espacio o escenario de transformación relacional que Maturana propone para operar cambios en la educación pasa necesariamente por el aprendizaje de la convivencia en armonía. Se trata de tolerar que las diferencias entre los seres humanos no residan en la inteligencia, en la capacidad de consenso, sino en las emociones, en lo que nos gusta, en aquello que disfrutamos y con lo cual experimentamos goce y despertar a nuevas realidades y conexiones para el equilibrio social y la felicidad individual.

Por todas las preocupaciones anteriores nos preguntamos ¿En qué consiste el surgimiento de la nueva consciencia? Existen respuestas fundamentales de pensadores como Erwin Lazslo (2004), quien nos expresa que "hay que estar claros que los cambios solamente resultan del nuevo estado mental e implican una conciencia planetaria" (p. 54).

Estamos conscientes que nos hace falta estar en acción, emocionarnos para iniciar a crear nuestro propio mapa que nos conduzca a tener una visión integral, vinculando la cultura de la sabiduría, la ciencia, el arte, tradiciones que nos permita reconstruir y construir nuestras vidas, mejorar la acción pedagógica en el proceso de aprendizaje, partiendo del rescate, las prácticas y saberes de los ancestros que por muchos años han mantenido las poblaciones indígenas en el buen vivir. Es un camino que debemos recorrer de manera que nos lleve a repensar nuestros actos, comportamientos, forma de vida, de consumo y de convivencia.

González de Alba (2002), considera que la ciencia actual nos ha conducido por un callejón sin salida y que "la actitud científica ha de ser reconstruida, que la ciencia ha de rehacerse de nuevo". Lo cual se relaciona con las proposiciones de Capra (1992), quién resalta que se deben reexaminar en profundidad las premisas y los valores más importantes de nuestra cultura, rechazando los modelos conceptuales anticuados e inútiles y recuperando otros valores descartados en los períodos precedentes de nuestra historia cultural.

Reflexionamos sobre la urgencia del cambio de paradigmas que es el principal motivo de este nuevo aprendizaje; necesitamos transitar del paradigma mecanicista a un paradigma holístico, fundamentado en la teoría del todo; conscientes que debemos generar cambios profundos de pensamientos que permitan realizar transformaciones sociales, económicas, políticas, culturales y educativas.

Por lo que Cosachov (2000), nos invita bajo el ala del arte, la alquimia y lo sagrado, una guía para intentar el cambio en diversas formas de búsqueda y nos da la imagen de hacer un mapa que nos lleve a la reflexión y análisis crítico de los nuevos modelos de formación humana y la realidad en que vivimos.

El paradigma emergente requiere de cambios humanos, que vibren desde nuestro interior, permitiendo hacer consciencia humana hacia la transformación de esquemas de pensamientos y de comprensión de la realidad, de las formas de relacionarnos con el entorno, con nuestros semejantes y con todo lo existente, de cambios en las sensibilidades y comprometernos por una sociedad mejor.

Bohm (1996:11), nos insta al diálogo, que cada persona tome consciencia de las creencias que operan en el grupo, incluyendo las propias, de manera que esto nos proporcione una nueva comprensión del carácter fragmentario y autodestructivo de muchos de nuestros pensamientos que sólo nos lleva a debilitar nuestras actitudes defensivas e infundir, de este modo, una cordialidad y un compañerismo connaturales al grupo. 
Los valores, la espiritualidad, la democracia tienen que ver con la biología del amor y sólo se mencionan o se hace reflexión sobre ellos en la medida en que estos son negados o se requiere destacarlos, porque en la cotidianidad son permanentemente negados.

\section{Responsabilizándonos del compromiso eco-pedagógico}

La naturaleza de la transformación en comunidad de acuerdo con Bohm, realizado desde el aprendizaje colectivo no sólo es posible, sino vital para realizar los potenciales de la inteligencia humana y para ello contamos con el diálogo a fin de explorar asuntos complejos de que todos los miembros de un grupo puedan aprender. Así, se logra desarrollar una sensibilidad que trasciende el acto de pensar y dado que los diálogos son creativos hacen surgir nuevas ideas.

Por lo anterior, retomamos la propuesta del diálogo que según Bohm conduce a las personas a expresar la sabiduría interior e invitando no sólo a la interiorización personal sino a la relación interpersonal y la integración de redes de ideas creativas que permiten la convivencia armónica.

La población autóctona de la Costa Caribe nicaragüense convive en un entramado de interconexiones desde la vida cotidiana, la recreación, el trabajo y la vida en general relacionada con la naturaleza, la organización social, el saber de sí mismo y la espiritualidad; herencia histórica generacional que ha venido transmitiéndose por tradición oral y prácticas cotidianas de la cultura. Es en este aspecto donde nuestra responsabilidad debe abonar, por una comprensión más profunda de aprendientes y la comunidad en general, a fin de lograr un aprendizaje para la ecología y el buen vivir.

La eco-educación para las comunidades de aprendizaje, deberá centrarse en la formación humana integral, para la motivación, por la alegría de vivir y el placer de relacionarse; producir a partir del despliegue de la inteligencia mediada, desarrollar el diálogo personal y comunitario que despierte la percatación de la eco-pedagogía logrando replantear una nueva visión del mundo, la naturaleza y el cosmos y aprovechar las facultades y fortalezas de las reservas mentales postergadas de las que nos habla Lozanov, afirmando además que la educación es lo más importante del mundo porque la vida entera es aprendizaje y conscientes de nuestro poten- cial tenemos la responsabilidad de no permanecer indiferentes sintiendo el llamado personal y grupal transformándonos para esta convivencia comunitaria y ejercer algún impulso del efecto mariposa para el interior de la región, el país y el mundo.

Este es el enfoque que se propone en estas reflexiones, por lo que se trata de auscultar en nuestros ambientes locales. Nos asomamos como miembros de la Costa Caribe a las comunidades diversas del territorio y descubrimos que poseen aprendizajes espontáneos para la vida grupal y allí se estimula el intercambio de saberes acumulados en la experiencia y en la memoria para la transmisión, compartimiento de habilidades, técnicas ancestrales, innovaciones para enfrentar la incertidumbre de su fragilidad climática o geográfica. Toda suerte de conocimientos y de medicina alternativa y por supuesto sus mitos y prácticas cargadas de espiritualidad donde integran rituales y danzas.

Es pertinente y justo mencionar que Nicaragua ha sido uno de los primeros países en el contexto centroamericano, en garantizar un ámbito de uso en la educación formal, para lenguas minoritarias a nivel nacional. El Ministerio de Educación consciente que la niñez de los pueblos miskitus, sumus- mayangna, ramas, krioles y garífunas; en la razón de que el español no es su lengua materna, al iniciar sus estudios al igual que sus docentes, se interrumpe la comunicación dificultando el proceso de aprendizaje; por esta razón se implementó la Educación Intercultural Bilingüe, a fin de responder a las necesidades educativas y de desarrollo de las Regiones Autónomas, a través de la creación del Programa de Educación Bilingüe Intercultural (PEBI).

El Programa de Educación Bilingüe Intercultural ha sido concebido y puesto en marcha, para ofrecer atención educativa en sus lenguas nativas a pueblos indígenas y afrodescendientes, contribuyendo al rescate cultural de las lenguas nativas para la inserción intercultural en la sociedad. En el caso de los indígenas mayangnas de la Costa Caribe Nicaragüense, cuya lengua ocupa un lugar especial en la ecología lingüística del Caribe, siendo amenazada no solamente por el español, sino por el miskitu. Este grupo indígena han contado con el apoyo de lingüistas internacionales en la documentación de su lengua y en la formación de especialistas de la lengua, para el rescate y revitalización de la misma. En este sentido nos enorgullece este proyecto promovido por la Universidad 
de las Regiones Autónomas de la Costa Caribe Nicaragüense (URACCAN), a cargo del Instituto para la Promoción Lingüística y Rescate Cultural (IPILC).

\section{Reconstrucción y empoderamiento social desde la psiquis colectiva}

Es la dimensión psíquica la que nos abre la fuente de todo cambio, Bohm considera a la consciencia como un fenómeno emergente construido en el ser humano, de igual manera lo hace Maturana planteando que esta estructura es siempre el resultado de las historias, en lo cual estos cambios han sido congruentes con las permutas estructurales del medio; pues todo ser vivo se encuentra en su presente y como resultado de esa historia vive de hecho en una continua transformación. Este es el fenómeno que se opera actualmente en nuestras consciencias y en los razonamientos individuales y grupales, con la expectativa de aplicación en nuestro entorno: familia y comunidad.

En esta nueva era del siglo XXI, debemos hacer un repaso de lo que hemos logrado en nuestro accionar con sus errores, debilidades y reflexionar sobre cuál será nuestra orientación en esta etapa, considerando esta inquietud, es oportuno explorar la situación de manera integral dándole una re-significación a la educación.

Cuando aprendemos se produce un cambio en el sentir, observar y actuar; cuando organizamos y modificamos nuestros pensamientos, consideramos fundamental el valor de la experiencia personal lo cual incide en el aprendizaje colectivo, dándole valor al ejemplo en la praxis.

Cuando reconocemos la validez del otro u otra, abrimos la posibilidad para que ese otro u otra exista en plenitud, valore su experiencia, contexto, historia, forma de ver y percibir la realidad en que vivimos; por esto estamos convencidos que la biopedagogía se caracteriza no por darnos respuestas, sino preguntas abiertas, para que cada una de las personas nos construyamos. En esto consiste la reconstrucción y el empoderamiento social; de esta manera ejercemos la ciudadanía que es la apropiación del derecho individual y colectivo. Varela (2002), lo plantea como la vinculación del conocimiento de la vida por medio de la consciencia del ser vivo, es decir, sentir, percibir, emocionar y razonar lo que nos permite construir un mundo enmarcado en la cognición o proceso del conocimiento, con el proceso de la vida.

Estas reflexiones nos llevan a nuevas formas de comprensión, diálogo, concertación, consenso, nuevos aprendizajes que nos permitan declararnos en búsqueda de esas nuevas formas de relación en la convivencia y el entorno con la aceptación y aprobación comunitaria que es donde reside la fuerza de la auto organización y donde experimentamos placer, pasión y emoción por lo que aprendemos y obtenemos en la vida; siempre que haya sido producto del ajuste fino de las energías y de los procesos de reconstrucción psíquica para integrar e interrelacionarnos con nuestro propio crecimiento, generando resonancia en nuestro entorno inmediato.

Debemos el ethos que nos permite amarnos y cuidarnos dentro de una cultura de paz que potencialice el gozo, el placer, el amor y la ternura, incluso elevarnos hacia la trascendencia suprema. El maestro Leonardo Boff considera que "Por la espiritualidad se capta a Dios como presencia inefable que se revela y vela en todos los procesos y que habla en la profundidad humana bajo forma de entusiasmo, de capacidad de amor, de perdón, de compasión y de veneración ante el misterio del universo". Este paso o escala de crecimiento humano se deriva inexorablemente de la reconstrucción psíquica individual y comunitaria que hacen posible potencializar las actitudes y valores de los miembros de la comunidad, con la intervención mediadora de aprendizajes y el objetivo de realizar la sanación de los estados de la psiquis.

Veamos el planteamiento visionario de Maturana (2002) para la interpretación de los factores incidentes en la transformación "Nuestra visión de la educación cognitiva, tiene una visión de que los humanos están cultural y genéticamente dotados e inclinados a ser aprendedores así como transformadores y generadores de información, teniendo por lo tanto el potencial para ser participantes activos y beneficiarios de la edad de la información" (p. 41).

Se necesita educar hombres y mujeres sensibles, profundamente humanos, capaces de cumplir compromisos, lo cual implica educar desde otro sentido, desde la ética tan necesaria en una sociedad que se mueve contraria a los valores morales y hacia la deshumanización del hombre" 
(Rigoberta Menchu, premio Nobel de la paz, 1992)

Esta cita nos lleva a entender el compromiso personal, grupal y comunitario, para que la educación sea factor determinante en la trasformación social que se viene señalando. Nos llama a realizar el cambio fundamental en el plano de nuestras creencias y consciencia; son ellas las que determinaran el mundo que habitamos. Necesitamos dar respuestas comunitarias a esta realidad; desde la conmoción que produce esta cruda realidad del planeta que agoniza ante el despale indiscriminado, la contaminación y la violencia social.

Todo este aprendizaje, nos conduce a desarrollar una espiritualidad con visión holista, a interrelacionarnos en igualdad con todos los seres vivientes en su diversidad, practicar la reciprocidad para llegar a una interdependencia voluntaria, asumiendo la ética cósmica como responsabilidad humana, buscando la complementariedad en la diversidad, tomando las decisiones en consenso, viviendo una economía comunitaria no acumulativa, entrando en sintonía con los ciclos de la naturaleza y del cosmos, respetando la sacralidad de toda la vida, como señala Gutiérrez (s/f),

Concebir el universo como lo hace la física moderna como una red de relaciones intrínsecamente dinámica, es uno de los aspectos esenciales de la cosmovisión de los pueblos primitivos que nos está urgiendo a la reconciliación de los seres humanos y de estos con el cosmo (p.7).

Una de las prácticas sociales que emplea la humanidad para empoderarse es la capacidad de manifestar sus ideas, sentimientos, pensamientos entre otros aspectos más es la comunicación. Podemos reconocer que entre las características de las formas de vivencia de la Costa Caribe Nicaraguense, uno de los principales valores de empoderamiento de ciudadanía, se evidencia en la forma de organización social y política que parte de los conocimientos ancestrales y el trabajo que se realiza de manera colectiva.

El contacto directo con la población autóctona de la Costa Caribe de Nicaragua, ha permitido evidenciar que los conocimientos están unidos a la vida cotidiana y al trabajo, relacionados a la naturaleza, la organización social y el saber de sí mismo. Hemos aprendido, que la convivencia en la diversi- dad, se sustenta en la ética del cosmos, basado en el principio que toda vida debe contribuir a la formación de una red de interrelaciones que posibiliten el inicio de la comunidad cósmica. Cuando damos y recibimos, establecemos relaciones sistemáticas que nos engrandece, gozamos, disfrutamos y nos humanizamos. En la visión indígena, es la manera de garantizar el equilibrio económico y ecológico. La reciprocidad no es considerada como obligación con la que uno debe corresponder matemáticamente con la misma medida, sino, es un querer corresponder en la misma forma que recibió en forma de don para mantener y consolidar la unidad, se necesita una actitud ética, flexible e inclusiva.

Debemos aprender, que la convivencia en la diversidad, se sustenta en la ética del cosmos, basado en el principio que toda vida debe contribuir a la formación de una red de interrelaciones que posibiliten el inicio de la comunidad cósmica. Cuando damos y recibimos, establecemos relaciones sistemáticas que nos engrandece, gozamos, disfrutamos y nos humanizamos.

Para concluir, es de notable interés valorar las ideas de Varela y Maturana sobre la historia natural de la reflexividad y conceptos de Ética con Biología y Pedagogía para describir que la tolerancia y el pluralismo, nos liberan de nuestros propios antivalores y percepciones si somos capaces de respetar las percepciones y los valores de los demás esto es una fórmula de solución para la superación del eterno conflicto entre los seres humanos, porque somos susceptibles al cambio y transformación en la convivencia gracias al amor que llevamos intrínseco en nuestra propia naturaleza.

\section{Conclusiones}

Si el propósito fundamental de la educación es el desarrollo humano, la convivencia armónica es lo fundamental para el enriquecimiento y profundización de las relaciones con nuestro yo interno, la familia, los miembros de la comunidad y con la comunidad global.

La eco-pedagogía enfatiza en la conexión y dependencia mutua de la naturaleza con la vida y la cultura humana y esto conduce hacia la libertad generándose la armonía, donde el ser humano encuentre el equilibrio, la equidad, la justicia y res- 
peto por el otro y la otra que ha sido la aspiración permanente de todos los tiempos.

Nos reconocemos como seres sociales, vinculados entre sí que ascendemos desde nuestros antepasados, con una herencia genética de experiencias a las que debemos dar continuidad e interacción para fortalecer nuestra propia existencia, con verdadero sentido de identidad, que en el caso nuestro nos pudimos ver de cuerpo entero, dentro de la realidad y circunstancias de la Costa Caribe Nicaragüense.

\section{Lista de referencias}

Boff, Leonardo (2002). Espiritualidad: Un camino de transformación. 3a Edición. Editorial Sal Terrae. España.

Bohm, David (1980). La Totalidady El orden implicado. Editorial Kairos, Barcelona.

Capra, Fritjof (1996). La trama de la vida. Una nueva perspectiva de los sistemas vivos. Editorial Anagrama, S.A. Barcelona.
Cosachov, M. (2000). Entre el cielo y la tierra Un viaje por el mapa del conocimiento. Buenos Aires: Biblos.

Gutiérrez, F. (s/f). Sentido del discurso pedagógico. Sin país.

González de Alba, Luis (2002). El burro de Sancho $y$ el gato de Schrodinger. Editorial Paidós Mexicana, S.A. México.

Payán de la Roche, Julio César. (200o). Lánzate al vacio, se extenderán tus alas. Santa $\mathrm{Fe}$ de Bogotá, D. C., Colombia: Mc Graw-Hill Interamericana, S. A.

Lazlo, Ervin (2004). La Ciencia y el Campo Akásico. Ediciones Nowtilus, S.L. España. Pág. 12.

Lozanov, (2000). Aprendizaje Acelerado: Estrategia para la potencialización del aprendizaje, Linda Kasuga y colaboradores México. Grupo Editorial, 2000.

Maturana, Humberto (2002). Transformación en la convivencia. 2a edición. DOLMEN EDICIONES, S.A.

Varela, Francisco (2004). El fenómeno de la vida. DOLMEN Ediciones. Caracas- MontevideoSantiago de Chile. 